\title{
Genipin Reverses HFD-Induced Liver Damage and Inhibits UCP2-Mediated Pyroptosis in Mice
}

\author{
Hong Zhong a,c Mengting Liu ${ }^{\mathrm{a}}$ Yaya Jia Minjuan Ma ${ }^{\mathrm{a}}$ Kun Chen ${ }^{\mathrm{d}}$ \\ Tingming Liang ${ }^{a}$ Chang Liu ${ }^{a, b}$
}

ajiangsu Key Laboratory for Molecular and Medical Biotechnology, School of Life Sciences, Nanjing Normal University, Nanjing, bSchool of Life Science and Technology, China Pharmaceutical University, Nanjing, 'Nanjing Maternity and Child Health Care Institute, Nanjing Maternity and Child Health Care Hospital, Obsterics and Gynecology Hospital Affiliated to Nanjing Medical University, Nanjing, dThe Joint Research Center of Guangzhou University and Keele University for Gene Interference and Application, School of Life Sciences, Guangzhou University, Guangzhou, China

\section{Key Words}

Liver damage • NAFLD • Genipin • Pyroptosis • UCP2

\begin{abstract}
Background/Aims: Liver damage is a typical manifestation of nonalcoholic fatty liver disease (NAFLD). It originates from excessive fat accumulation, leading to hepatocyte death, inflammation, and fibrosis. Nonalcoholic steatohepatitis (NASH) is a type of NAFLD with a prevalence of $49 \%$ in morbidly obese patients. Pyroptosis plays an important role in the development of $\mathrm{NASH}$; thus, it is important to elucidate the effect of lipid accumulation on pyroptosis. Genipin (GNP), a natural water-soluble cross-linking agent, has hepatoprotective effects and decreases lipid accumulation in the liver; however, the mechanisms underlying these effects are unknown. Methods: In this study, qPCR and Western blot were used to examine pyroptotic gene expression in high-fat diet (HFD) induced obese mice and free fatty acids (FFAs) treated hepatocytes. At the same time, relative lactate dehydrogenase (LDH) release and Hoechst \& propidium iodide (PI) staining were done to verify cell death. To explore the molecular mechanism, cell transfection were constructed with siRNA or plasmid to obtain knockdown or overexpression hepatocytes. Results: We found that HFD-fed mice and FFAs-treated hepatocytes had obvious pyroptosis, and addition of GNP reversed liver damage and inhibited pyroptosis both in vitro and in vivo. Besides, UCP2 knockdown cells showed suppressed FFAs-mediated pyroptosis, as determined by decreased pyroptotic gene expression, reduced lactate dehydrogenase (LDH) release, and reduced cell death. Consistent with this, cells transfected with UCP2 had upregulated pyroptotic gene expression, increased
\end{abstract}




\section{Cellular Physiology Cell Physiol Biochem 2018;49:1885-1897

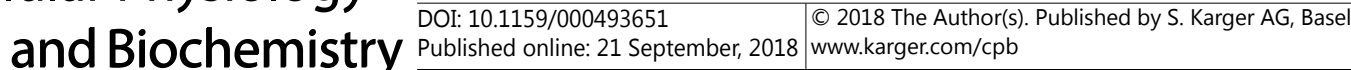 \\ Zhong et al.: Genipin Alleviates Liver Damage and Pyroptosis in Mice}

LDH release, and increased cell death. Conclusion: GNP reverses HFD-induced liver damage and inhibits UCP2-mediated pyroptosis. Thus, GNP may serve as a potential therapeutic candidate for NAFLD.

\section{Introduction}

Liver damage, a typical manifestation of nonalcoholic fatty liver disease (NAFLD), is the hepatic counterpart of metabolic disorders, ranging from hepatic steatosis to nonalcoholic steatohepatitis (NASH), fibrosis, cirrhosis, and hepatocellular carcinoma [1]. NAFLD originates from excessive fat accumulation in liver cells, leading to hepatocyte death, inflammation, and fibrosis [2]. With improvements in living standards and an increase in sedentary behavior, the prevalence of NAFLD has been gradually increasing, affecting $20-30 \%$ of the general population and $75-100 \%$ of obese individuals [3]. In general, the prevalence of NASH among patients with NAFLD is $10 \%-20 \%$, of whom $8 \%-25 \%$ progress to liver cirrhosis. In addition, up to $2.8 \%$ of patients with NASH develop end-stage liver disease or hepatocellular carcinoma [4]. The prevalence of NASH is as high as $49 \%$ in morbidly obese NAFLD patients [5]. Scientists have paid close attention to remedy NAFLD. Zeng et al. demonstrated that Alisol A 24-acetate effectively ameliorated hepatic steatosis and inhibited inflammation [6]. Another study also reported that taurine play protective role of in NAFLD via FGF-21/SIRT1 axis [7]. However, applications of these drugs are in low efficiency. Thus, it is important to elucidate the pathophysiology of liver damage during the progression of $\mathrm{NASH}$, and to develop effective therapies for the growing NAFLD epidemic.

Pyroptosis, a new type of programmed cell death originally defined as a caspase- 1 (Casp1)-dependent modality, can be triggered by the infection of bacterial pathogens (Salmonella typhimurium, Shigella flexneri, Listeria monocytogenes, and Pseudomonas aeruginosa), viral toxins (lipopolysaccharide [LPS], Bacillus anthracis) or disease (stroke, cancer), accompanied by cytoskeletal rearrangement, pore formation in the plasma membrane, DNA fragmentation, and release of pro-inflammatory and pyrogenic cytokines [8]. Following pore formation, small molecular weight dyes such as propidium iodide (PI) become permeable to the plasma membrane, while apoptotic cells remain intact and cannot be stained [9]. At the same time, the penetrable plasma membrane allows ions and water to rush into the cell, resulting in rupture of swollen cells and release of intracellular content. Thus, lactate dehydrogenase (LDH), a type of cytosolic enzyme in the cell, can be detected in the extracellular milieu [10]. These characteristics are sufficient to identify pyroptosis.

The molecular determinants and upstream signals of pyroptosis are delicate. Casps are cysteine proteases that use active-site cysteine residues to cleave target proteins after aspartate residues, and play central roles in initiating apoptosis and inflammatory pyroptosis. Apoptotic Casps include initiator Casps (Casps 2, 8, 9, and 10) and effector Casps (Casps 3, 6, and 7) that orchestrate apoptotic cell death. In contrast, inflammatory pyroptotic Casps (Casps 1, 4, 5 in humans and murine Casp11) are crucial mediators of inflammation and cell death [11]. Casp1 exists in inactive pro-forms in the cytosol and is activated when cleaved into p10 and p20 catalytic domain fragments [12], which is required for interleukin 1 beta (IL-1 $\beta$ ) processing, IL-18 release, and induction of pyroptosis. Casp1 is activated by the canonical Nod-like receptors (NLRs, typically via their caspase recruitment domain [CARD] or Pyrin domain), such as through direct CARD-CARD interactions (e.g., NLRP1b and NLRC4) or apoptosis-associated speck-like protein containing a CARD (ASC, also known as PYCARD)mediated interactions (e.g., NLRP3, AIM2 and Pyrin) [13]. ASC is only composed of a Pyrin and CARD domain, and is an adapter protein for Casp1 activation [14]. It can interact with itself to create a cascade effect, recruiting all ASCs to a single subcellular location termed the "ASC focus" or "pyroptosome" [15]. Gasdermin D (GSDMD), recently identified as a generic substrate for Casp1 and executioner in pyroptosis [16], plays different roles in canonical and non-canonical inflammasomes. In canonical pathways, activated Casp1 cleaves GSDMD into a $31 \mathrm{kDa}$-terminal fragment to drive pyroptosis. In non-canonical pyroptosis, LPS resides 


\section{Cellular Physiology Cell Physiol Biochem 2018;49:1885-1897

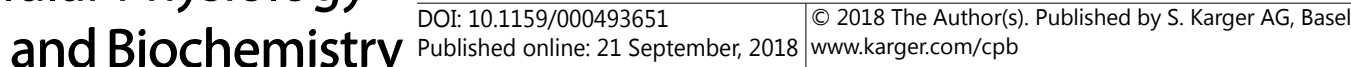 \\ Zhong et al.: Genipin Alleviates Liver Damage and Pyroptosis in Mice}

in the cytoplasm and binds to Casps 4, 5, and 11 to release the cleaved N-terminal domain and initiate pyroptosis [17].

Accumulating evidence has suggested that pyroptosis plays a critical role in metabolic diseases. Wree et al. demonstrated that global or to a lesser extent myeloid-specific activation of NLR family pyrin domain containing 3 (NLRP3) results in hepatocyte pyroptosis, severe liver inflammation, and fibrosis [18]. Another study found that aberrant or excessive activation of Casp1 plays an important role in promoting survival upon pathogen attack and regulating lipid metabolism [19]. It was also shown that Casp1 knockout mice did not release IL-1 $\beta$ and IL-18, and were resistant to endotoxic shock [20]. Importantly, keeping donor organs in the cold and flushed with a solution containing IDN-65561773 to inhibit Casp1 activity, provided $40 \%$ protection against ischemia-reperfusion liver injury, as indicated by decreased serum alanine aminotransferase (ALT) levels, and also ameliorated neutrophil accumulation in the liver 7 days after liver transplantation [21]. In addition, Casp1 is activated in macrophages and hepatocytes during the development of NASH, suggesting its important role in inflammation and fibrosis during NASH development [22, 23]. A recent study showed that GSDMD plays a key role in the pathogenesis of NASH in humans and mice [24], broadening the possibilities for NASH therapy.

Mitochondrial dysfunction appears at different levels of NASH pathogenesis, presenting ultrastructural mitochondrial alterations, impairment of hepatic ATP synthesis, and the increased production of reactive oxygen species [25]. Moreover, overexpression of uncoupling protein-2 (UCP2) in the liver can cause acute liver injury [26]. The natural product genipin (GNP), an inhibitor of UCP2, was shown to enhance ATP production in isolated pancreatic islets and improve mitochondrial function in mice [27, 28]. UCP2 also suppresses inflammation and inhibits NLRP3 expression [29, 30]. In addition, UCP2 knockout mice were completely resistant to Toxoplasma gondii infection, suggesting that UCP2 has an antiinflammatory role [31]. Therefore, we investigated the role of UCP2 in the progression of pyroptosis.

We found that high-fat diet (HFD)-fed obese mice and free fatty acids (FFAs)-treated hepatocytes showed obvious pyroptosis. Administration of GNP reversed HFD-induced liver damage and inhibited pyroptosis through UCP2. These results demonstrated that UCP2 overexpression enhanced pyroptosis. Thus, GNP may serve as a potential therapeutic target for liver disease.

\section{Materials and Methods}

\section{Animals}

All animal experiments were conducted according to the Guide for the Care and Use of Laboratory Animals published by the National Institutes of Health (NIH publication 85-23, revised in 1996) and the accepted rules enacted by the Laboratory Animal Care Committee at Nanjing Normal University (Permit 2090658, issued 20 April 2008). Male C57BL/6J mice aged 6-8 weeks purchased from the Model Animal Research Center of Nanjing University (Nanjing, Jiangsu, China), and were housed in the animal facilities with a $12: 12$-h light/dark cycle, controlled temperature $\left(22-24^{\circ} \mathrm{C}\right)$ and humidity $(50-60 \%)$, for 1 week quarantine with free access to water and food. Subsequently, mice were randomly divided into two groups: 20 mice were given an ND (10\% calories from fat, 20\% calories from protein, 70\% calories from carbohydrate; Research Diets Inc., New Brunswick, NJ, USA) and 30 were fed with a HFD (60\% calories from fat, $20 \%$ calories from protein, $20 \%$ calories from carbohydrate; Research Diets). After 2 months, an obese mouse model with significant steatohepatitis was obtained. Five different treatment protocols were administered by gavage (10 mice per group): 1) ND with normal saline (ND+Saline); 2) ND with $20 \mathrm{mg} /$ $\mathrm{kg} / \mathrm{d}$ GNP (ND+GNP [20 mg/kg]); 3) HFD with normal saline (HFD+Saline); 4) HFD with $5 \mathrm{mg} / \mathrm{kg} / \mathrm{d}$ GNP (HFD+GNP [5 mg/kg]); and 5) HFD with $20 \mathrm{mg} / \mathrm{kg} / \mathrm{d}$ GNP (HFD + GNP [20 mg/kg]). GNP (Zelang, Nanjing, Jiangsu, China) was dissolved in normal saline. 


\section{Cellular Physiology Cell Physiol Biochem 2018;49:1885-1897 \begin{tabular}{l|l|l} 
and Biochemistry 10.1159/000493651 & $\begin{array}{l}\text { C) } 2018 \text { The Author(s). Published by S. Karger AG, Basel } \\
\text { www.karger.com/cpb }\end{array}$ \\
\hline
\end{tabular}

\section{Liver histochemical staining}

After treated with GNP or saline for 12 weeks, mice were fasted for $2 \mathrm{~h}$ and sacrificed to harvest their liver tissues. Small pieces of liver were cut and submerged in $4 \%$ paraformaldehyde, embedded in paraffin, sectioned into $5-\mu \mathrm{m}$ thick sections, and stained with H\&E. Images were taken with an inverted microscope (Nikon Eclipse Ts2R; Nikon, Tokyo, Japan ).

\section{Biochemical analysis}

Hepatic ALT and AST levels were measured with commercially available test kits (Jiancheng Institute of Biotechnology, Nanjing, Jiangsu, China). Simultaneously, the protein concentrations in cell lysates were measured by the BCA Protein Assay method (Jiancheng Institute of Biotechnology). The enzyme activity was exhibited as units per gram of protein (U/g protein).

\section{Cell culture and treatments}

AML12 hepatocytes were cultured in a 1:1 mixture of Dulbecco's Modified Eagle's Medium and Ham's F12 medium (DMEM/F12, Wisent, St. Bruno, Canada) supplemented with $10 \%$ fetal bovine serum (FBS, Wisent), 100 units $/ \mathrm{mL}$ penicillin (Sigma-Aldrich, St. Louis, MO, USA), $100 \mathrm{mg} / \mathrm{mL}$ streptomycin sulfate (Sigma), $0.005 \mathrm{mg} / \mathrm{mL}$ insulin, $0.005 \mathrm{mg} / \mathrm{mL}$ transferrin, $5 \mathrm{ng} / \mathrm{mL}$ selenium (ITS, Crygen, Nottingham, UK), and $40 \mathrm{ng} / \mathrm{mL}$ dexamethasone (Sigma). Cells were placed in a humidified atmosphere containing 5\% CO2 at $37^{\circ} \mathrm{C}$ at a density of $2.5 \times 105$ or $5 \times 104$ cells per well on 6 -well or 24 -well plates, respectively. For stimulation, reagents were added to the medium without FBS after fasting for $6 \mathrm{~h}$. GNP was dissolved in dimethyl sulfoxide and FFAs were prepared by adding equal amounts of oleic acid and palmitic acid with $12.5 \%$ BSA.

\section{Plasmid construction and siRNA transfection}

The complete coding sequence of the mouse UCP2 gene (930 bp) was amplified from the cDNA with the following primers:

(forward)5'-CCGGAATTCATGGTTGGTTTCAAGGCC-3';

(reverse) 5'-CCGCTCGAGTCAGAAAGGTGCCTCCC-3', cleaved by EcoR1 and Xho1 restriction enzymes, and inserted into the pcDNA 3.0 vector. siUCP2 was produced by RiboBio (Guangzhou, Guangdong, China). Overexpression or knockdown of UCP2 was induced by transfection of $500 \mathrm{ng}$ plasmid or $100 \mathrm{nM}$ siRNA, respectively, using Lipofectamine 3000 (Thermo Fisher Scientific, Waltham, MA, USA), according to the manufacturer's instructions.

\section{Relative LDH release}

After various treatments, cells were centrifuged at $1000 \mathrm{rpm}$ for $2 \mathrm{~min}$, after which relative LDH release was determined using a commercial kit (Beyotime, Shanghai, China) according to the manufacturer's instructions. The absorbance of samples was assessed at $490 \mathrm{~nm}$ using a microplate reader (Epoch; Biotek, Winooski, VT, USA).

\section{Staining assay}

To evaluate pore formation in the cell membrane, Hoechst and PI (Beyotime) were used for staining at $37^{\circ} \mathrm{C}$ in the dark. Following the appropriate treatment, cells were stained with Hoechst for $10 \mathrm{~min}$. After washing three times with phosphate-buffered saline, PI was added for another $10 \mathrm{~min}$. Cells with blue and red fluorescence were observed using a fluorescence microscope (Nikon Eclipse Ts2R).

\section{Quantitative RT-PCR}

cDNA obtained by reverse transcription of $1 \mu \mathrm{g}$ RNA was extracted using TRIzol reagent (Invitrogen, Carlsbad, CA, USA) in liver tissues, and gene expression was analyzed in the hepatocytes. The primers used were as follows (5' to 3' direction):

Gsdmd (forward) CCATCGGCCTTTGAGAAAGTG; (reverse) ACACATGAATAACGGGGTTTCC;

Casp1 (forward) ACAAGGCACGGGACCTATG; (reverse) TCCCAGTCAGTCCTGGAAATG;

Ucp2 (forward) GCGTTCTGGGTACCATCCTA; (reverse) GCTCTGAGCCCTTGGTGTAG;

36B4 (forward) GAAACTGCTGCCTCACATCCG; (reverse) GCTGGCACAGTGACCTCACACG.

36B4 was used to normalize mRNA levels. 


\section{Cellular Physiology Cell Physiol Biochem 2018;49:1885-1897

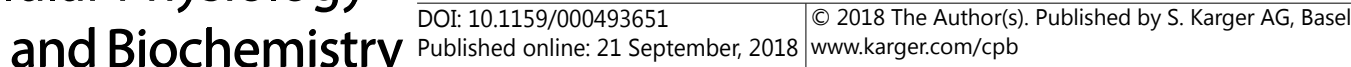 \\ Zhong et al.: Genipin Alleviates Liver Damage and Pyroptosis in Mice}

\section{Western blotting}

Cells or liver tissues were lysed in RIPA buffer (Roche, Basel, Switzerland) and PMSF (Beyotime). Then the samples were incubated overnight with GSDMD (Santa Cruz, Dallas, TX, USA), CASP1 (Proteintech, Wuhan, China), UCP2 (Proteintech), and $\beta$-Actin (Origin, Wuhan, China) antibodies. Images were taken using the Tanon 5200 system (Tanon Science \& Technology, Shanghai, China). Protein quantification was performed using AlphaEascFC (FluorChem 8800 imaging system; Alpha Innotech Co., San Jose, CA, USA). These experiments were performed in triplicate.

\section{Statistical analysis}

Data are presented as the mean \pm standard deviation from multiple samples. All experiments were repeated at least three times. Group differences were considered statistically significance at $p<0.05$ assessed by the Student's t-test.

\section{Results}

Pyroptosis is induced in HFD-induced obese mice and FFAs-treated hepatocytes

Because pyroptosis results in liver damage and HFD-fed mice show obvious steatohepatitis, we used these models to determine whether pyroptosis is activated by excess lipid. After mice were fed a HFD for 5 months, we collected the liver tissues and examined the molecular markers of pyroptosis. The mRNA expression of Gsdmd and Casp1 increased by 1.5- and 2.2-fold, respectively (Fig. 1A). At the same time, the protein content of GSDMD-N and p20, active effectors of GSDMD and CASP1, respectively were also enhanced, indicating the induction of pyroptosis in HFD-fed mice (Fig. 1B, C). Interestingly, the mRNA and protein hepatic expression of Ucp2, a gene located in the mitochondrial inner membrane that is related to obesity-related fatty liver, was also significantly increased (Fig. 1A-C). To confirm these observations, we treated AML12 mouse hepatic cells with gradient concentrations of FFAs for different time points to analyze the role of lipid for pyroptosis in vitro, as both oleate and palmitate can induce lipotoxicity [32]. Unexpectedly, the expression of Ucp2 and pyroptotic-related genes were not induced in time- or dose-dependent manners. The optimum condition was treatment with $1 \mathrm{mM}$ FFAs for $6 \mathrm{~h}$ (Fig. 1D-I). In addition, LDH released into the medium was significantly elevated at the course (Fig. 1J). However, Hoechst and PI staining showed that the number of pyroptotic cells was increased by FFAs treatment (Fig. 1K).

\section{GNP reverses liver damage and inhibits pyroptosis in obese mice}

GNP is a natural compound with a protective role against liver disease. By hepatic hematoxylin and eosin (H\&E) staining, we found that HFD-fed mice had severe lipid accumulation and inflammation. Notably, GNP significantly reduced the amount of lipid droplets and inflammation sites in a dose-dependent manner (Fig. 2A). Consistently, hepatic ALT and aspartate aminotransferase (AST), markers of liver injury, were also decreased in obese mice treated with GNP, whereas levels in normal diet (ND) mice were unchanged (Fig. $2 \mathrm{~B}, \mathrm{C})$. At the molecular level, GNP decreased the mRNA expression of inflammatory markers such as tumor necrosis factor alpha (TNF- $\alpha$ ), IL-1 $\beta$, and IL-6, which was increased by a HFD (Fig. 2D). Furthermore, we GNP decreased Ucp2 expression in normal and obese mice, while pyroptotic gene expression was only decreased in HFD-fed mice (Fig. 2E). The protein content of all markers was consistent with the mRNA levels (Fig. 2F, G). Together, these data demonstrate the inhibitory role of GNP in liver damage and pyroptosis.

GNP inhibits pyroptosis in hepatocytes

Next, we examined the effects of GNP in vitro. In cultured AML12 hepatocytes, GNP only decreased Ucp2 expression, whereas the expression of pyroptotic-related genes remained unchanged (Fig. 3A-C). Interestingly, GNP decreased the protein levels of active GSDMD-N and p20 in FFAs-treated cells by $25 \%$ and $34 \%$, respectively (Fig. 3B, C). We monitored relative 


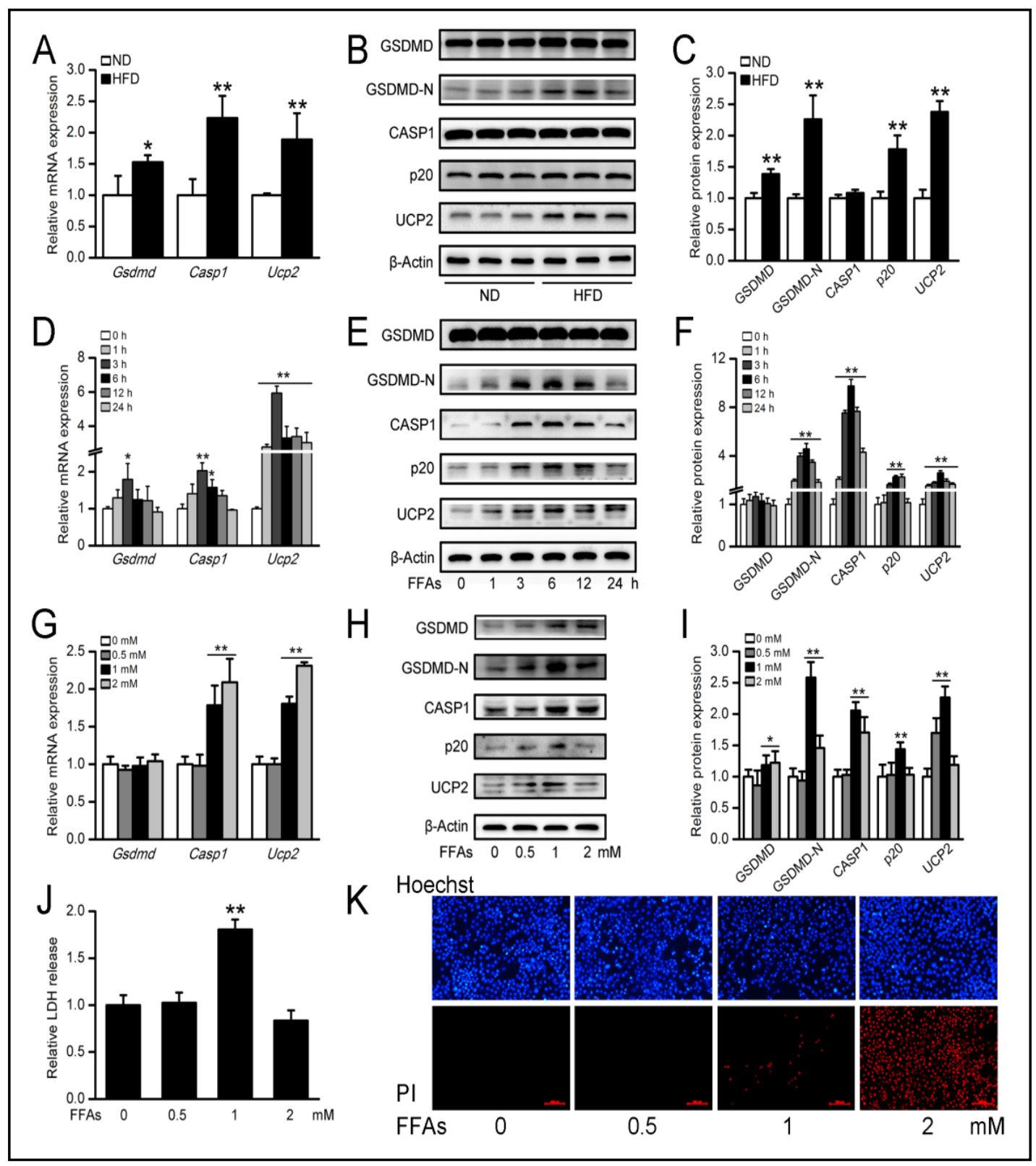

Fig. 1. Pyroptosis is induced by FFAs in mice and hepatocytes. (A) Relative mRNA expression in the liver of mice. The protein content levels assayed by Western blot were shown in (B) images and (C) densitometric determinations. Data were shown as mean $\pm S D, n=6$ mice/group. ${ }^{*} p<0.05$ and ${ }^{* *} p<0.01$ vs ND.(D) Relative mRNA expression in AML12 cells treated with 1 mM FFAs for different time. The protein content was shown in (E) images and (F) densitometric determinations. (G) Relative mRNA expression in AML12 cells treated with different concentrations of FFAs for $6 \mathrm{~h}$. The protein content was shown in (H) images and (I) densitometric determinations. (J) Relative LDH release in AML12 cells treated with different concentrations of FFAs for 6 h. (K) Hoechst and PI staining in AML12 cells treated with different concentrations of FFAs for $6 \mathrm{~h}$. Experiments were repeated in triplicate and data were shown as mean $\pm S D,{ }^{*} p<0.05$ and ${ }^{* *} \mathrm{p}<0.01$ vs control. 


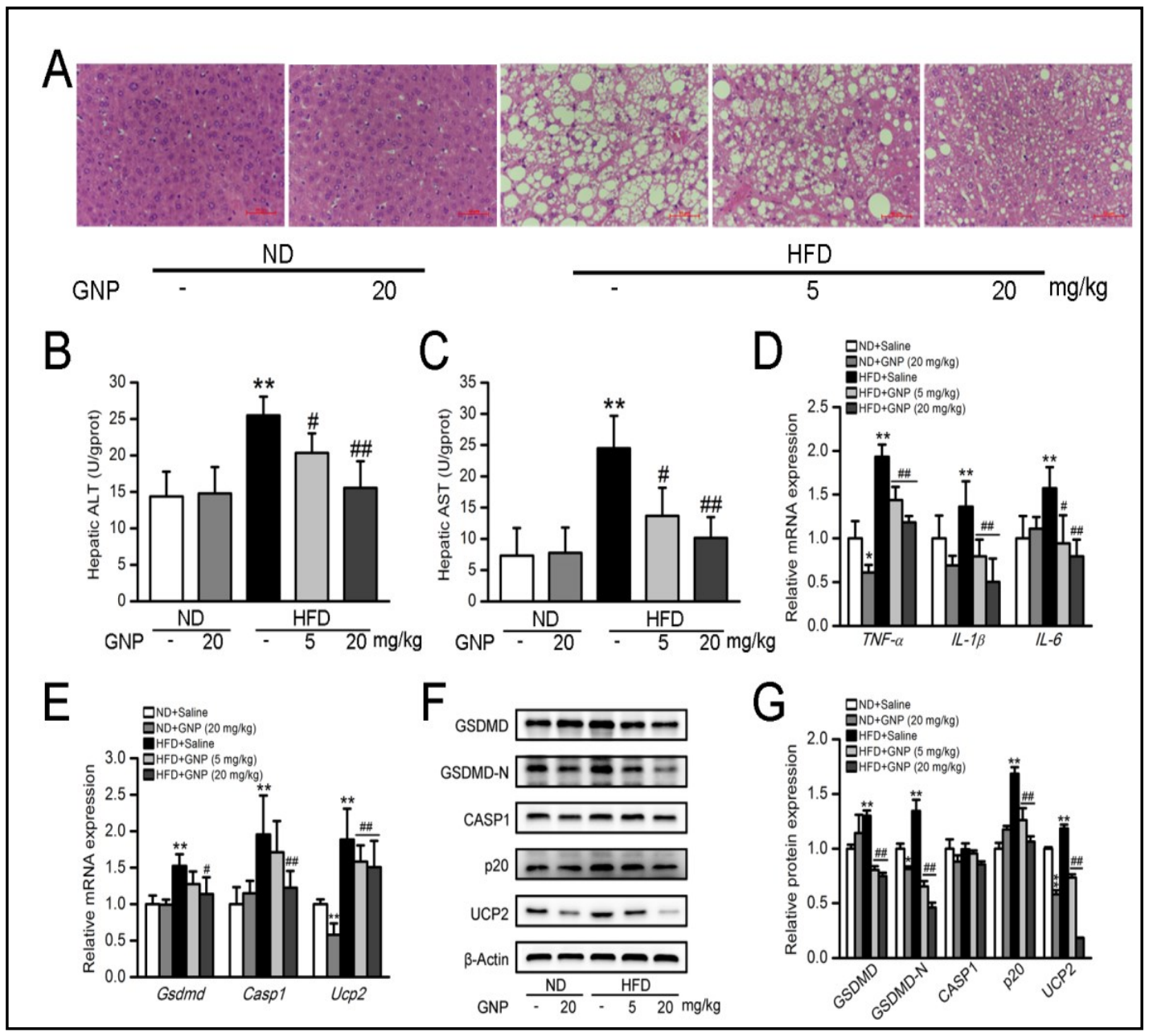

Fig. 2. GNP protects mice from HFD-induced liver injury and pyroptosis. (A) Liver tissue sections stained with H\&E to reveal lipid droplets and inflammation. Bars $=100 \mu \mathrm{m}$. (B) Hepatic ALT and (C) AST were determined by commercial kits to show liver injury. (D) Relative mRNA expression of genes related to inflammation. (E)Relative mRNA expression in mice treated with GNP. The protein content was shown in (F) images and (G) densitometric determinations. Data were shown as mean \pm SD, $n=6$ mice/group. ${ }^{*} \mathrm{p}<0.05$ and ${ }^{* *} \mathrm{p}<0.01$ vs ND; ${ }^{\#}<0.05$ and ${ }^{\# \#} \mathrm{p}<0.01$ vs HFD.

LDH release in cells treated with FFAs or GNP, and found that LDH release was increased by 1.8-fold with FFAs, and was reduced by GNP (Fig. 3D), indicating the inhibitory effects of GNP in FFAs-induced pyroptosis. Cell staining showed that FFAs increased the number of pyroptotic cells, but supplementation with GNP had preventative effects, as determined by the decrease in cell death (Fig. 3E).

\section{Pyroptosis induced by FFAs is mediated by UCP2}

Because GNP, an inhibitor of UCP2, suppressed pyroptosis in vivo and in vitro, we determined if its inhibitory effects in pyroptosis were through UCP2. To test our hypothesis, we reduced Ucp2 expression using small interfering RNA (siRNA). The knockdown efficiencies at the mRNA and protein levels were $50 \%$ and $18 \%$, respectively (Fig. 4A-C). Upon treatment with GNP, the FFAs-mediated protein expression of active GSDMD-N and p20 and release of LDH was suppressed in siUCP2 cells (Fig. 4B-D). Consistently, the number of pyroptotic cells was decreased in siUCP2 cells (Fig. 4E). Together, these findings demonstrate that UCP2 mediated induction effect of FFAs on pyroptosis. 


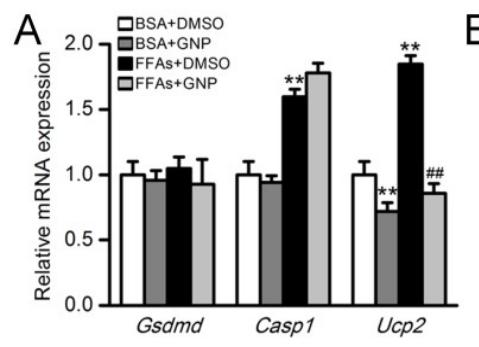

$B$

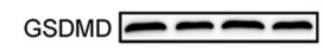
GSDMD-N $==\equiv$
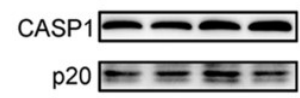
UCP2 $-\cdots$

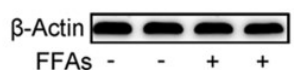
$\begin{array}{lll}\text { FFAs }-- & + & + \\ \text { GNP }-+ & + & +\end{array}$
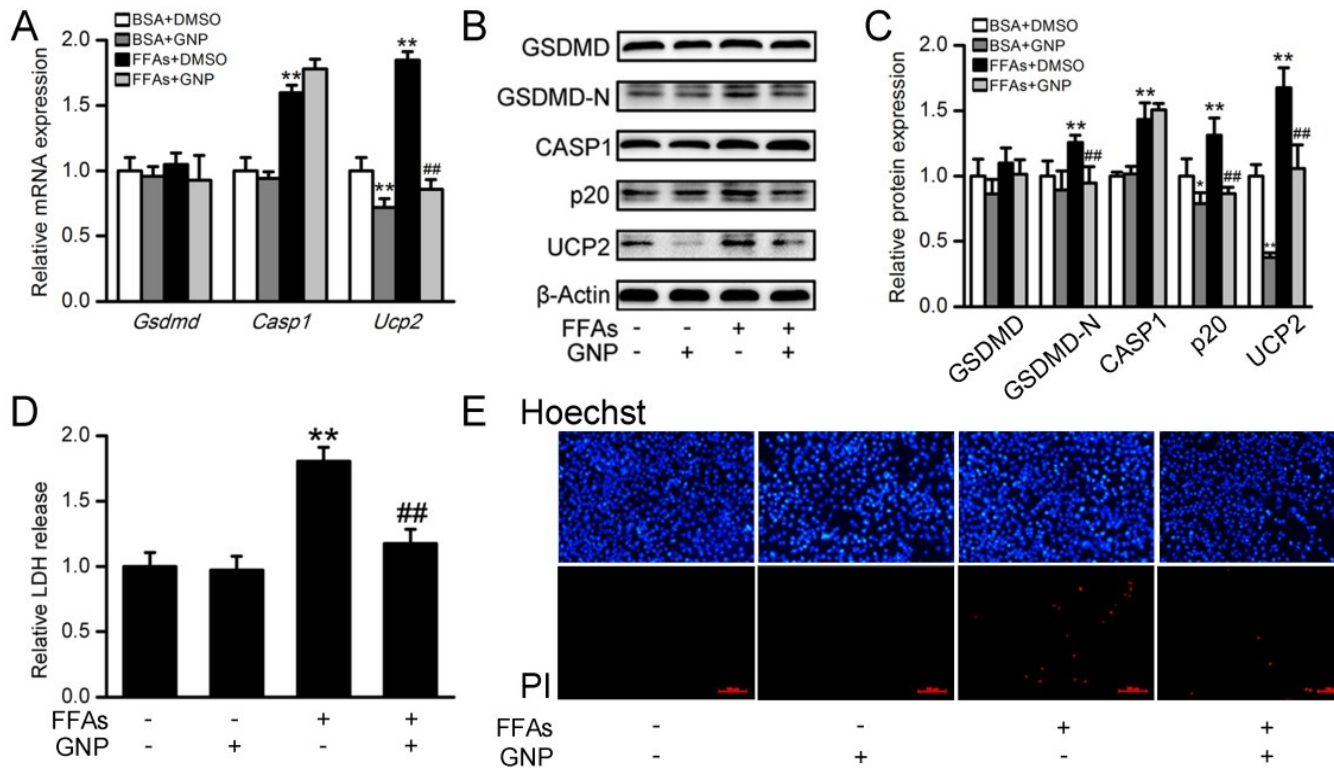

E Hoechst

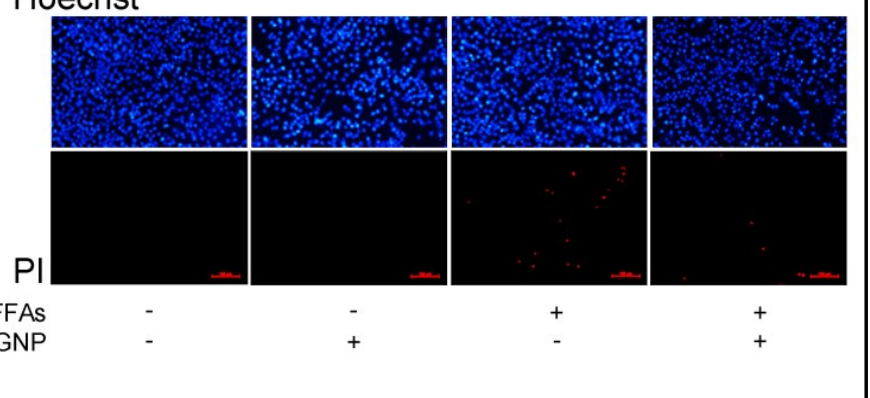

Fig. 3. GNP treatment prevents pyroptosis in hepatocytes. (A) Relative mRNA expression in AML12 cells treated with $1 \mathrm{mM}$ FFAs or $50 \mu \mathrm{M}$ GNP for $6 \mathrm{~h}$. The protein content was shown in (B) images and (C) densitometric determinations. (D) Relative LDH release in AML12 cells treated with 1 mM FFAs or $50 \mu \mathrm{M}$ GNP for $6 \mathrm{~h}$. (E) Hoechst and PI staining in AML12 cells treated with $1 \mathrm{mM}$ FFAs or $50 \mu \mathrm{M}$ GNP for $6 \mathrm{~h}$. Experiments were repeated in triplicate and data were shown as mean $\pm S D,{ }^{*} p<0.05$ and ${ }^{* *} \mathrm{p}<0.01$ vs BSA+DMSO; ${ }^{\# \#} \mathrm{p}<0.01$ vs FFAs+DMSO.

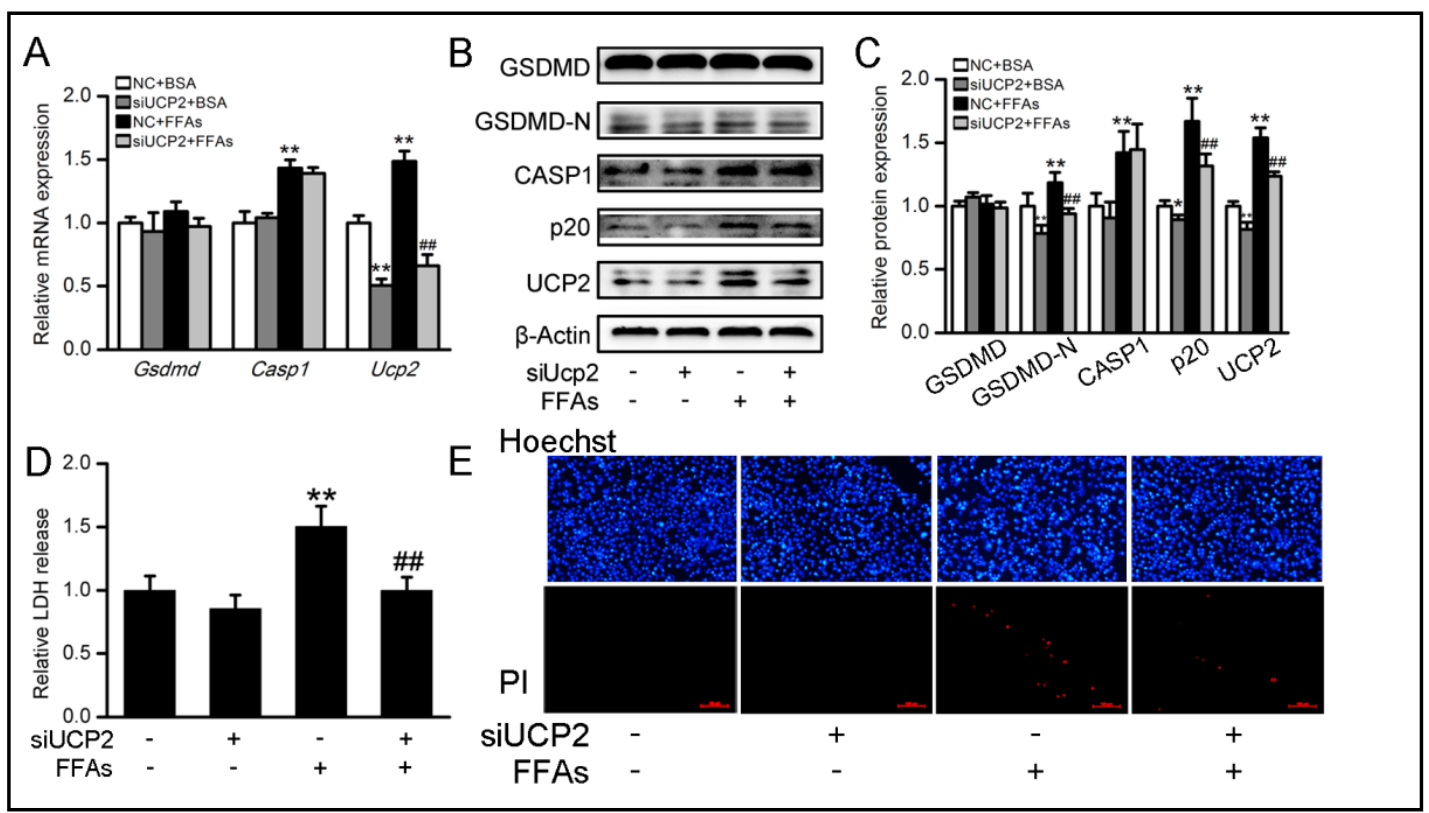

Fig. 4. Pyroptosis induced by FFAs is mediated by Ucp2. Cells were transfected with $100 \mathrm{nM}$ siRNAs to knockdown UCP2 expression for $36 \mathrm{~h}$. Subsequently, $1 \mathrm{mM}$ FFAs were supplemented to the cells after fasted for 6 h. (A) Relative mRNA expression in AML12 cells with various treatments. The protein content was shown in (B) images and (C) densitometric determinations. (D) Relative LDH release in AML12 cells. (E) Hoechst and PI staining in AML12 cells. Experiments were repeated in triplicate and data were shown as mean \pm SD, ${ }^{*} p<0.05$ and ${ }^{* *} \mathrm{p}<0.01$ vs $\mathrm{NC}+\mathrm{BSA}$; ${ }^{\# \#} \mathrm{p}<0.01$ vs $\mathrm{NC}+\mathrm{FFAs}$. 


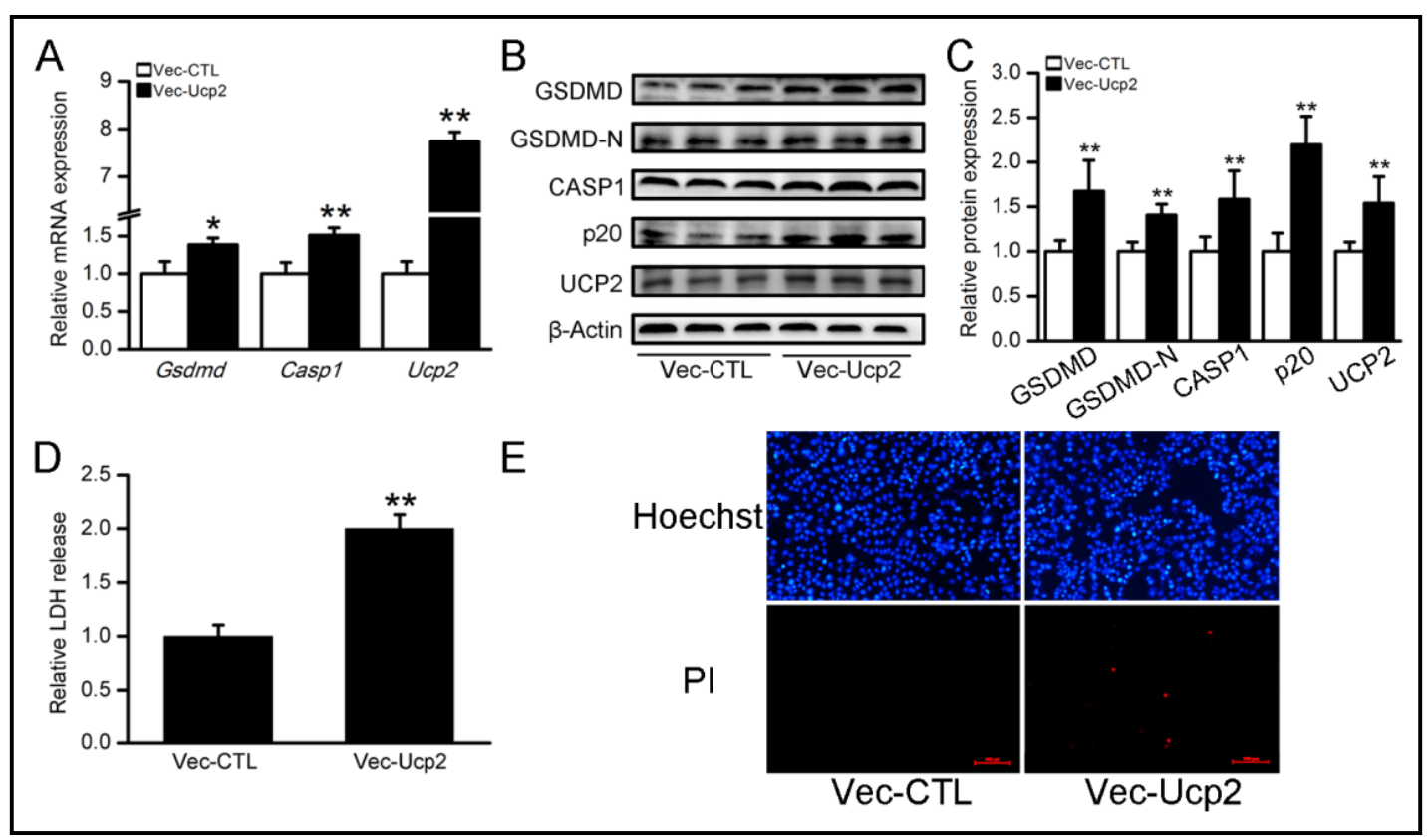

Fig. 5. Pyroptosis is activated by Ucp2 in hepatocytes. Cells were transfected with $500 \mu \mathrm{g}$ constructed plasmid to overexpress UCP2 expression for $36 \mathrm{~h}$. (A) Relative mRNA expression in AML12 cells with various treatments. The protein content was shown in (B) images and (C) densitometric determinations. (D) Relative LDH release in AML12 cells. (K) Hoechst and PI staining in AML12 cells. Experiments were repeated in triplicate and data were shown as mean $\pm \mathrm{SD},{ }^{*} \mathrm{p}<0.05$ and ${ }^{* *} \mathrm{p}<0.01$ vs Vec-CTL.

\section{UCP2 is essential for pyroptosis}

To explore the role of UCP2 in pyroptosis, we constructed a vector with the complete coding sequence of UCP2 and transfected it into AML12 cells. Ucp2 was overexpressed by 7.5fold compared to the control, and relative Gsdmd and Casp1 expression was also significantly induced (Fig. 5A). At the protein level, UCP2 was increased by 1.5 -fold, and GSDMD and CASP1 expression was increased by 1.7- and 1.6-fold, respectively. The expression of active GSDMD-N and p20 was increased by 1.4- and 2.2-fold, respectively (Fig. 5B, C). LDH release was also upregulated by 2 -fold after transfection with UCP2 plasmid (Fig. 5D). Hoechst and PI staining showed an increase in pyroptotic cells in UCP2-overexpressing cells (Fig. 5E), suggesting the essential role of UCP2 in pyroptosis.

\section{Discussion}

In this study, we found that HFD-fed mice showed obvious pyroptosis in the liver, which was induced by FFAs accumulation in AML12 hepatocytes. GNP, a natural compound, played an important role in protecting against liver damage by inhibiting pyroptosis. At the molecular level, knockdown and overexpression experiments showed that the effects of GNP on pyroptosis were mediated by UCP2. Specifically, GNP reversed HFD-induced liver damage and ameliorated pyroptosis through UCP2, which has not been previously reported, suggesting the preventative role of GNP against hepatic disease.

Many pathways function in the pathogenesis of NAFLD, one of the alarmingly problems in the 21st century [33]. Hepatocytes derived factor S1P is activated by palmitate and released into the extracellular environment to involve in the progression of liver fibrosis [34]. Besides, miR-122 and miR-155 also play important role in triglyceride accumulation in the liver $[35,36]$. Further studies are needed to classify the detailed mechanisms.

Apoptosis, necrosis, and pyroptosis, different types of intrinsic cell death mechanism are induced by intracellular pathogens. Apoptosis is active, programmed cell death that

\section{KARGER}




\section{Cellular Physiology Cell Physiol Biochem 2018;49:1885-1897

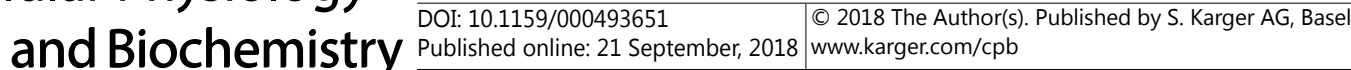 \\ Zhong et al.: Genipin Alleviates Liver Damage and Pyroptosis in Mice}

does not elicit inflammation, while necrosis is characterized by passive, accidental death with the release of inflammatory intracellular contents. Pyroptosis was recently found to be a form of programmed cell death, which is initiated by inflammasomes and executed by GSDMD, resulting in pore formation and cell rupture. Unfortunately, pyroptosis kills infected host cells but does not damage the intracellular pathogens [37]; thus, it is more effective in defense against environmental stimulus. GSDMD has binding affinity for lipids that usually reside in the cytosolic leaflet of the plasma membrane and protects adjacent cells from pyroptosis [38]. Our observation that HFD-fed mice and FFAs-treated hepatocytes showed obvious pyroptosis (Fig. 1) is in accordance with those findings.

Pyroptosis is transiently induced as the pore formation breaks the normal permeability barrier and initiates compensatory mechanisms by activating ion channels or emergency exocytic membrane fusion events to patch the plasma film $[39,40]$. In this study, pyroptoticrelated genes were initially upregulated, but returned to baseline in AML12 cells treated with FFAs for different time points (Fig. 1D-F). UCP2 expression was regulated in the same manner in accordance with a previous study, which showed that the strongest UCP2 activation was in hepatoma cells treated with palmitic acid for $6 \mathrm{~h}$ [41]. In our study, the optimum dose for induction of pyroptosis was 1 mM FFAs; higher doses led to maximum cell death accompanied by a decrease in pyroptosis-related gene expression and LDH release (Fig. 1G-K). The observed cell death with decreased pyroptosis was due to other cell death mechanisms such as necrosis.

GNP, the metabolite of geniposide, is the major active form present in fruit of Gardenia jasminoides, and possesses cholagogue, anti-inflammatory, anti-tumor, and anti-oxidative effects. Numerous studies have shown its hepatoprotective effects in liver disease. For example, Lin et al. [42] reported that treatment of NAFLD patients with GNP and vitamin E markedly relieved adipose degeneration in liver cells. Qiu et al. found that GNP ameliorated podocyte injury in diabetic mice through inhibition of UCP2, indicating the important role of this gene [43]. Takeuchi et al. [44] found that GNP improved acute liver dysfunction by suppressing TNF- $\alpha$ production. In our previous study, we also found that GNP alleviated hepatic lipid accumulation via the miR-142a/SREBP-1c axis [45]. Collectively, these data show that GNP has therapeutic benefits in patients with liver disease. Interestingly, another study showed that inhibition of UCP2 with GNP exacerbated palmitate-induced hepatic steatosis, which is controversial with previous study demonstrating decreased triacylglycerol concentrations [46], may be attributed to the low concentration $(5 \mu \mathrm{M})$ and the compensatory effect of upregulated UCP2 expression.

Recentstudies have shown that GNP inhibits NLRP3 and NLRC4 inflammasome activation [47], which is responsible for hepatocyte pyroptosis, liver inflammation, and fibrosis. Thus, in this study, we explored the effects of GNP on lipid accumulation-induced liver damage and pyroptosis. We found that GNP reversed HFD-fed-induced liver damage and inhibited inflammatory cytokine production (Fig. 2A-D). Our in vitro and in vivo studies demonstrated that GNP inhibited pyroptosis induced by FFAs (Fig. 2E-G and 3). It is well known that GNP is an inhibitor of UCP2; thus we hypothesized that the effects of GNP in mediating FFAsinduced pyroptosis was via UCP2. To test this hypothesis, we constructed UCP2 knockdown cells and found that the induction effect of FFAs on pyroptosis was suppressed, as determined by decreased pyroptotic-related gene expression, reduced LDH release, and less cell death (Fig. 4). In contrast, cells transfected with UCP2 had upregulated pyroptotic-related gene expression and increased LDH release and cell death. Therefore, we conclude that UCP2 is essential for pyroptosis. Of note, pyroptosis remained unchanged in normal cells treated with GNP or transfected with siRNA, suggested that it could only be reverted after induction by FFAs. However, it is unlikely that the pyroptosis-suppressing effects of GNP are solely via inhibition of UCP2 expression; thus, further studies are needed to identify the mechanisms underlying the role of GNP in pyroptosis. 


\section{Cellular Physiology Cell Physiol Biochem 2018;49:1885-1897 \begin{tabular}{ll|l} 
and Biochemistry & $\begin{array}{l}\text { DOI: 10.1159/000493651 } \\
\text { Published online: } 21 \text { September, } 2018\end{array}$ & $\begin{array}{l}\text { 2018 The Author(s). Published by S. Karger AG, Basel } \\
\text { www.rom/cpb }\end{array}$ \\
\cline { 2 - 3 }
\end{tabular} \\ Zhong et al.: Genipin Alleviates Liver Damage and Pyroptosis in Mice}

\section{Conclusion}

In conclusion, we found that GNP effectively reversed HFD-induced liver damage and inhibited pyroptosis through UCP2, thereby providing an additional therapeutic strategy for the growing NAFLD epidemic.

\section{Abbreviations}

ALT (alanine aminotransferase); AST (aspartate aminotransferase); CARD (caspase recruitment domain); Casp1 (caspase-1); FFAs (free fatty acids); GNP (genipin); GSDMD (gasdermin D); H\&E (hematoxylin and eosin); HFD (high-fat diet); IL (interleukin); LDH (lactate dehydrogenase); NAFLD (nonalcoholic fatty liver disease); NASH (nonalcoholic steatohepatitis); ND (normal diet); NLRs (Nod-like receptors); PI (propidium iodide); SD (standard deviation); UCP2 (uncoupling protein-2).

\section{Disclosure Statement}

The authors have no conflicts of interest to declare.

\section{Acknowledgements}

This work was supported by grants from the National Natural Science Foundation (NSF) of China $(31422028,31771298,31401009)$, the NSF of Jiangsu Province of China (Grant No. BK20140041), National Basic Research Program of China (973 Program) (2012CB947600, 2013CB911600), the Collaborative Innovation Center for Cardiovascular Disease Translational Medicine (Nanjing Medical University), the Priority Academic Program Development of Jiangsu Higher Education Institutions, and the Innovation Project of Nanjing Normal University (184080H10369).

\section{References}

1 Gaemers IC, Groen AK: New insights in the pathogenesis of non-alcoholic fatty liver disease. Curr Opin Lipidol 2006;17:268-273.

-2 Angulo P: Nonalcoholic fatty liver disease. N Engl J Med 2002;346:1221-1231.

-3 Henao-Mejia J, Elinav E, Jin C, Hao L, Mehal WZ, Strowig T, Thaiss CA, Kau AL, Eisenbarth SC, Jurczak MJ, Camporez JP, Shulman GI, Gordon JI, Hoffman HM, Flavell RA: Inflammasome-mediated dysbiosis regulates progression of NAFLD and obesity. Nature 2012;482:179-185.

4 Lee J, Kim Y, Friso S, Choi SW: Epigenetics in non-alcoholic fatty liver disease. Mol Aspects Med 2017;54:7888.

5 Campos GM, Bambha K, Vittinghoff E, Rabl C, Posselt AM, Ciovica R, Tiwari U, Ferrel L, Pabst M, Bass NM, Merriman RB: A clinical scoring system for predicting nonalcoholic steatohepatitis in morbidly obese patients. Hepatology 2008;47:1916-1923.

-6 Zeng L, Tang W, Yin J, Feng L, Li Y, Yao X, Zhou B: Alisol A 24-Acetate Prevents Hepatic Steatosis and Metabolic Disorders in HepG2 Cells. Cell Physiol Biochem 2016;40:453-464.

7 Abd Elwahab AH, Ramadan BK, Schaalan MF, Tolba AM: A Novel Role of SIRT1/ FGF-21 in Taurine Protection Against Cafeteria Diet-Induced Steatohepatitis in Rats. Cell Physiol Biochem 2017;43:644-659.

8 Kepp 0, Galluzzi L, Zitvogel L, Kroemer G: Pyroptosis - a cell death modality of its kind? Eur J Immunol 2010;40:627-630.

-9 Silveira TN, Zamboni DS: Pore formation triggered by Legionella spp. is an Nlrc4 inflammasome-dependent host cell response that precedes pyroptosis. Infect Immun 2010;78:1403-1413. 


\section{Cellular Physiology Cell Physiol Biochem 2018;49:1885-1897 \begin{tabular}{l|l|l} 
and Biochemistry & Dublished online: 21 September, 2018 & $\begin{array}{l}\text { C } 2018 \text { The Author(s). Published by S. Karger AG, Basel } \\
\text { www.karger.com/cpb }\end{array}$
\end{tabular}

10 Fink SL, Cookson BT: Caspase-1-dependent pore formation during pyroptosis leads to osmotic lysis of infected host macrophages. Cell Microbiol 2006;8:1812-1825.

11 Siegel RM: Caspases at the crossroads of immune-cell life and death. Nat Rev Immunol 2006;6:308-317.

12 Broz P, von Moltke J, Jones JW, Vance RE, Monack DM: Differential requirement for Caspase-1 autoproteolysis in pathogen-induced cell death and cytokine processing. Cell Host Microbe 2010;8:471483.

13 Jimenez Fernandez D, Lamkanfi M: Inflammatory caspases: key regulators of inflammation and cell death. Biol Chem 2015;396:193-203.

14 Schroder K, Tschopp J: The inflammasomes. Cell 2010;140:821-832.

15 Fernandes-Alnemri T, Wu J, Yu JW, Datta P, Miller B, Jankowski W, Rosenberg S, Zhang J, Alnemri ES: The pyroptosome: a supramolecular assembly of ASC dimers mediating inflammatory cell death via caspase-1 activation. Cell Death Differ 2007;14:1590-1604.

16 Shi J, Zhao Y, Wang K, Shi X, Wang Y, Huang H, Zhuang Y, Cai T, Wang F, Shao F: Cleavage of GSDMD by inflammatory caspases determines pyroptotic cell death. Nature 2015;526:660-665.

17 Man SM, Kanneganti TD: Gasdermin D: the long-awaited executioner of pyroptosis. Cell Res 2015;25:11831184.

18 Wree A, Eguchi A, McGeough MD, Pena CA, Johnson CD, Canbay A, Hoffman HM, Feldstein AE: NLRP3 inflammasome activation results in hepatocyte pyroptosis, liver inflammation, and fibrosis in mice. Hepatology 2014;59:898-910.

19 Gurcel L, Abrami L, Girardin S, Tschopp J, van der Goot FG: Caspase-1 activation of lipid metabolic pathways in response to bacterial pore-forming toxins promotes cell survival. Cell 2006;126:1135-1145.

20 Kayagaki N, Warming S, Lamkanfi M, Vande Walle L, Louie S, Dong J, Newton K, Qu Y, Liu J, Heldens S, Zhang J, Lee WP, Roose-Girma M, Dixit VM: Non-canonical inflammasome activation targets caspase-11. Nature 2011;479:117-121.

-21 Baskin-Bey ES, Washburn K, Feng S, Oltersdorf T, Shapiro D, Huyghe M, Burgart L, Garrity-Park M, van Vilsteren FG, Oliver LK, Rosen CB, Gores GJ: Clinical trial of the pan-caspase inhibitor, IDN-6556, in human liver preservation injury. Am J Transplant 2007;7:218-225.

-22 Dixon LJ, Berk M, Thapaliya S, Papouchado BG, Feldstein AE: Caspase-1-mediated regulation of fibrogenesis in diet-induced steatohepatitis. Lab Invest 2012;92:713-723.

-23 Csak T, Ganz M, Pespisa J, Kodys K, Dolganiuc A, Szabo G: Fatty acid and endotoxin activate inflammasomes in mouse hepatocytes that release danger signals to stimulate immune cells. Hepatology 2011;54:133-144.

-24 Xu B, Jiang M, Chu Y, Wang W, Chen D, Li X, Zhang Z, Zhang D, Fan D, Nie Y, Shao F, Wu K, Liang J: Gasdermin D plays a key role as a pyroptosis executor of non-alcoholic steatohepatitis in humans and mice. J Hepatol 2017;10.1016/j.jhep.2017.11.040.

25 Serviddio G, Sastre J, Bellanti F, Vina J, Vendemiale G, Altomare E: Mitochondrial involvement in nonalcoholic steatohepatitis. Mol Aspects Med 2008;29:22-35.

26 Shang Y, Liu Y, Du L, Wang Y, Cheng X, Xiao W, Wang X, Jin H, Yang X, Liu S, Chen Q: Targeted expression of uncoupling protein 2 to mouse liver increases the susceptibility to lipopolysaccharide/galactosamineinduced acute liver injury. Hepatology 2009;50:1204-1216.

-27 Guan L, Feng H, Gong D, Zhao X, Cai L, Wu Q Yuan B, Yang M, Zhao J, Zou Y: Genipin ameliorates age-related insulin resistance through inhibiting hepatic oxidative stress and mitochondrial dysfunction. Exp Gerontol 2013;48:1387-1394.

28 Zhang CY, Parton LE, Ye CP, Krauss S, Shen R, Lin CT, Porco JA, Jr., Lowell BB: Genipin inhibits UCP2mediated proton leak and acutely reverses obesity- and high glucose-induced beta cell dysfunction in isolated pancreatic islets. Cell Metab 2006;3:417-427.

29 Rajanbabu V, Galam L, Fukumoto J, Enciso J, Tadikonda P, Lane TN, Bandyopadhyay S, Parthasarathy PT, Cho Y, Cho SH, Lee YC, Lockey RF, Kolliputi N: Genipin suppresses NLRP3 inflammasome activation through uncoupling protein-2. Cell Immunol 2015;297:40-45.

30 Koo HJ, Song YS, Kim HJ, Lee YH, Hong SM, Kim SJ, Kim BC, Jin C, Lim CJ, Park EH: Antiinflammatory effects of genipin, an active principle of gardenia. Eur J Pharmacol 2004;495:201-208.

-31 Arsenijevic D, Onuma H, Pecqueur C, Raimbault S, Manning BS, Miroux B, Couplan E, Alves-Guerra MC, Goubern M, Surwit R, Bouillaud F, Richard D, Collins S, Ricquier D: Disruption of the uncoupling protein-2 gene in mice reveals a role in immunity and reactive oxygen species production. Nat Genet 2000;26:435439. 


\section{Cellular Physiology Cell Physiol Biochem 2018;49:1885-1897 \begin{tabular}{ll|l|l|l} 
DOI: 10.1159/000493651 & 2018 The Author(s). Published by S. Karger AG, Basel \\
and Biochemistry & Published online: 21 September, 2018 ww.com/cpb
\end{tabular}

-32 Hetherington AM, Sawyez CG, Zilberman E, Stoianov AM, Robson DL, Borradaile NM: Differential Lipotoxic Effects of Palmitate and Oleate in Activated Human Hepatic Stellate Cells and Epithelial Hepatoma Cells. Cell Physiol Biochem 2016;39:1648-1662.

33 Lou Y, Chen YD, Sun FR, Shi JP, Song Y, Yang J: Potential Regulators Driving the Transition in Nonalcoholic Fatty Liver Disease: a Stage-Based View. Cell Physiol Biochem 2017;41:239-251.

-34 Al Fadel F, Fayyaz S, Japtok L, Kleuser B: Involvement of Sphingosine 1-Phosphate in Palmitate-Induced Non-Alcoholic Fatty Liver Disease. Cell Physiol Biochem 2016;40:1637-1645.

-35 Wu GY, Rui C, Chen JQ, Sho E, Zhan SS, Yuan XW, Ding YT: MicroRNA-122 Inhibits Lipid Droplet Formation and Hepatic Triglyceride Accumulation via Yin Yang 1. Cell Physiol Biochem 2017;44:1651-1664.

-36 Wang L, Zhang N, Wang Z, Ai DM, Cao ZY, Pan HP: Decreased MiR-155 Level in the Peripheral Blood of Non-Alcoholic Fatty Liver Disease Patients may Serve as a Biomarker and may Influence LXR Activity. Cell Physiol Biochem 2016;39:2239-2248.

37 Jorgensen I, Rayamajhi M, Miao EA: Programmed cell death as a defence against infection. Nature Reviews Immunology 2017;17:151-164.

-38 Liu X, Zhang Z, Ruan J, Pan Y, Magupalli VG, Wu H, Lieberman J: Inflammasome-activated gasdermin D causes pyroptosis by forming membrane pores. Nature 2016;535:153-158.

39 Hoffmann EK, Lambert IH, Pedersen SF: Physiology of cell volume regulation in vertebrates. Physiological Reviews 2009;89:193-277.

40 McNeil PL, Kirchhausen T: An emergency response team for membrane repair. Nat Rev Mol Cell Biol 2005;6:499-505.

41 Lou J, Wang Y, Wang X, Jiang Y: Uncoupling protein 2 regulates palmitic acid-induced hepatoma cell autophagy. Biomed Res Int 2014;2014:810401.

-42 Lin L, Guan X, Wang L, Tang Y: Influence of genipin and vitamin E on UCP2 and other correlation factors in non-alcoholic fatty liver disease. Sheng Wu Yi Xue Gong Cheng Xue Za Zhi 2010;27:1327-1331.

-43 Qiu W, Zhou Y, Jiang L, Fang L, Chen L, Su W, Tan R, Zhang CY, Han X, Yang J: Genipin inhibits mitochondrial uncoupling protein 2 expression and ameliorates podocyte injury in diabetic mice. PLoS One 2012;7:e41391.

44 Takeuchi S, Goto T, Mikami K, Miura K, Ohshima S, Yoneyama K, Sato M, Shibuya T, Watanabe D, Kataoka E, Segawa D, Endo A, Sato W, Yoshino R, Watanabe S: Genipin prevents fulminant hepatic failure resulting in reduction of lethality through the suppression of TNF-alpha production. Hepatol Res 2005;33:298-305.

45 Zhong H, Chen K, Feng M, Shao W, Wu J, Chen K, Liang T, Liu C: Genipin alleviates high-fat diet-induced hyperlipidemia and hepatic lipid accumulation in mice via miR-142a-5p/SREBP-1c axis. FEBS J 2017;10.1111/febs.14349.

46 Kojima K, Shimada T, Nagareda Y, Watanabe M, Ishizaki J, Sai Y, Miyamoto K, Aburada M: Preventive effect of geniposide on metabolic disease status in spontaneously obese type 2 diabetic mice and free fatty acidtreated HepG2 cells. Biol Pharm Bull 2011;34:1613-1618.

-47 Yu SX, Du CT, Chen W, Lei QQ Li N, Qi S, Zhang XJ, Hu GQ, Deng XM, Han WY, Yang YJ: Genipin inhibits NLRP3 and NLRC4 inflammasome activation via autophagy suppression. Sci Rep 2015;5:17935. 